\title{
43. APORTACIONES AL ESTUDIO FITOSOCIOLÓGICO DE LAS COMUNIDADES DE BOJ (BUXUS SEMPERVIRENS) EN EL SECTOR SUBBÉTICO (ANDALUCÍA, ESPAÑA)
}

\author{
Juan Antonio TORRES, Eusebio CANO y Antonio GARCÍA FUENTES
}

Contribution to the phytosociological study of Buxus sempervirens communities in the Subbetic biogeographical Sector (Andalusia, Spain).

Palabras clave. Buxus sempervirens, Sector Subbético, Quercetea ilicis, fitosociología.

Key words. Buxus sempervirens, Quercetea ilicis, Subbético Sector, phytosociology.

En el presente trabajo pretendemos profundizar en el estudio de las comunidades de Buxus sempervirens presentes en el sector Subbético, en base a las investigaciones que actualmente realizamos en las sierras de Pandera y Alta Coloma (subsector Subbético Maginense).

Ecológicamente, tal y como indica Gómez Mercado et al. (1988), el boj (Buxus sempervirens) es una especie calcícola, de requerimientos hídricos elevados (subhúmedohúmedo), generalmente asociada a bosques caducifolios, con suelos profundos y humificados, por lo que se ha considerado como característica de Quercetalia pubescentis. No obstante, es fácil encontrarla en suelos esqueléticos, roquedos y taludes calizos, siempre y cuando se mantengan las condiciones mesofíticas. Se trata de una especie que presenta gran resistencia a los sustratos de esta naturaleza.

En los estudios sobre las comunidades de Buxus sempervirens realizados en los subsectores Cazorlense y Alcaracense, varios autores, Galiano (1960), Martínez Parras et al. (1976), Boucher (1984) y Valle (1985), señalan la relación existente entre el boj y los bosques de caducifolios de quejigares y acerales, pero sin presentar ninguno de ellos un encuadre fitosociológico definido.
Finalmente, Gómez Mercado et al. (1988) proponen tres tipos de bojedas para los subsectores Cazorlense y Alcaracense, aludiendo a la importancia de la naturaleza del suelo como elemento diferenciador de uno y otro tipo de bojeda: a) bojedas climáticas, ligadas a suelos profundos $\mathrm{y}$, por tanto, al bosque caducifolio Daphno latifoliae-Aceretum granatensis Rivas-Martínez 1964 subas buxetosum sempervirentis Gómez-Mercado \& Valle 1988 , b) topográficas o edafoxerófilas y ligadas a sabinares Junipero phoeniceaePinetum salzmanii Valle, Mota \& GómezMercado 1988 subas. buxetosum sempervirentis Gómez-Mercado \& Valle 1988 y c) higrófilas, en relación con la edafoserie riparia Lonicero splendidae-Buxetum sempervirentis GómezMercado \& Valle 1988.

Por nuestra parte, y en base a los estudios realizados en las sierras de Pandera y Grajales (subsector Subbético-Maginense), ampliamos el areal corológico de las bojedas climáticas Daphno latifoliae-Aceretum granatensis RivasMartínez 1964 subas. buxetosum sempervirentis Gomez-Mercado \& Valle 1988, así como el de las bojedas higrófilas Lonicero splendidae-Buxetum semervirentis Gómez Mercado \& Valle 1988, al subsector SubbéticoMaginense y proponemos una nueva subasociación de boj ligada a sabinares de 


\begin{tabular}{lrrrrrrrrr}
\hline & $\mathbf{1}$ & $\mathbf{2}$ & $\mathbf{3}$ & $\mathbf{4}$ & $\mathbf{5}$ & $\mathbf{6}$ & $\mathbf{7}$ & $\mathbf{8}$ & $\mathbf{9}$ \\
Altitud (m): & 1100 & 1000 & 1100 & 1000 & 1000 & 1100 & 1100 & 1150 & 1100 \\
Cobertura (\%): & 70 & 40 & 80 & 60 & 40 & 60 & 60 & 70 & 70 \\
Inclinación (\%): & 5 & 5 & 5 & 15 & 20 & 5 & 5 & 0 & 15 \\
Orientación: & $\mathrm{S}$ & $\mathrm{S}$ & $\mathrm{N}$ & $\mathrm{N}$ & $\mathrm{S}$ & $\mathrm{N}$ & $\mathrm{N}$ &. & $\mathrm{N}-\mathrm{E}$ \\
Altura media (m): & 1 & 3 & 3 & 1 & 3 & 2 & 1,5 & 3 & 3 \\
\hline
\end{tabular}

\section{Juniperus phoenicea Rhamnus lycioides Juniperus oxycedrus \\ Pinus halepensis \\ Quercus rotundifolia \\ Rubia peregrina \\ Daphne gnidium}

Diferencial de subasociación

Buxus sempervirens

Compañeras

$\begin{array}{lllllllll}. & 1 & 1 & 1 & 1 & 1 & 3 & 3 & 3 \\ + & + & + & 1 & + & + & + & + & 1 \\ . & . & + & 2 & 1 & + & . & . & + \\ + & . & . & + & . & . & . & . & + \\ + & 1 & 1 & + & 1 & + & . & 1 & 1 \\ . & . & + & . & . & . & . & . & . \\ . & + & + & . & . & . & + & + & .\end{array}$

$\begin{array}{lllllllll}3 & 2 & 4 & 3 & 2 & 3 & 3 & 2 & 2\end{array}$

\begin{tabular}{|c|c|c|c|c|c|c|c|c|}
\hline Echinospartum boissieri & 2 & + & + & 1 & 1 & 3 & 2 & + \\
\hline Thymus orospedanus & 1 & 2 & 1 & 2 & + & 1 & . & 1 \\
\hline Ulex parviflorus & 1 & + & 1 & + & . & . & 1 & 2 \\
\hline Rosmarinus officinalis & 1 & . & 1 & + & . & 1 & + & + \\
\hline Helianthemum cinereum ssp. rubellum & . & . & . & . & + & 1 & 1 & 1 \\
\hline Quercus faginea & . & + & . & . & + & . & . & + \\
\hline Amelanchier ovalis & . & . & . & . & + & . & . & . \\
\hline Iberis saxatilis ssp. saxatilis & + & + & . & . & . & . & . & . \\
\hline Spartium junceum & + & + & . & . & . & & . & \\
\hline Sangisorba minor & . & + & . & . & . & . & . & . \\
\hline Helianthemum croceum & . & . & + & + & . & . & + & . \\
\hline Cistus clussi & + & + & . & . & . & . & . & . \\
\hline Asphodelus albus & . & + & + & . & . & . & & . \\
\hline
\end{tabular}

Además: Helianthemum hirtum 1, Reseda lutea 2, Cistus albidus + en 1; Tulipa sylvestris ssp. australis + en 2; Bupleurum spinosum + en 4; Teucrium capitatum + en 6; Berberis hispanica + en 7; Andryala ragusina 1, Cynosurus elegans + , Rumex bucephalophorus 1, Crambe filiformis,+ Paronychia argentea + , Prunus spinosa 1 en 8; Thymus zygis 1, Bunium alpinum + en 9.

Localidades: 1; Inicio Barranco Los Cortijuelos. 2; Inicio Barranco Los Cortijuelos. 3; Próximo Barranco Los Cortijuelos. 4; Barranco afluente de B. Cornicabra. 5; Hacia la mitad de B. Los Cortijuelos. 6; Llano de Los Cortijuelos. 7; Laderas altas de B. Los Cortijuelos. 8; Alto cercano a Cortijo Los Cortijuelos. 9; Cerca Cortijo Los Cortijuelos. (Todos los inventarios realizados en la Sierra de Grajales, Carchelejo, Jaén).

Tabla 1. As. Rhamno lycioidis-Juniperetum phoeniceae Rivas Martínez \& López González in López González 1976 subas. buxetosum sempervirentis nova.

Juniperus phoenicea: Rhamno lycioidiJuniperetum phoeniceae Rivas Martínez \& López González in López González 1976 subas buxetosum sempervirentis nova que a continuación describimos.
Se trata de una bojeda topográfica o edafoxerófila, con una aceptable cobertura, que se instala sobre suelos poco evolucionados de tipo litosoles o roquedos de carácter básico, en el seno de los sabinares de Juniperus 
phoenicea (López, 1976), siempre que estos roquedos coincidan con barrancos 0 exposiciones umbrosas que permiten la existencia de una humedad ambiental elevada (subhúmedo-húmedo). La falta de suelo limita la evolución de estas formaciones hacia otras más mesofíticas, tal y como demuestra la presencia de Quercus faginea y Amelanchier ovalis en algunos inventarios de nuestra tabla.

La presencia de algunos endemismos béticos entre las especies compañeras, tal es el caso de Echinospartum boissieri y Thymus orospedanus, dan el matiz corológico a esta bojeda. Presenta su óptimo en el subsector Subbético-Maginense (sector Subbético), donde hemos realizado nuestros inventarios, en los pisos bioclimáticos mesomediterráneo superior y supramediterráneo bajo ombroclima subhúmedo (tab. 1, inv. 1 al 9, holotipo n 9).

Sintaxonómicamente, se incluye en la alianza indiferente edáfica Rhamno lycioidesQuercion cocciferae Rivas Goday \& Rivas Martínez 1975 del orden Pistacia-Rhamnetalia alaterni Rivas Martínez 1975, clase Quercetea ilicis Br.Bl. 1947.

\section{BIBLIOGRAFÍA}

BOUCHER, C. -1984-Contribution à l'étude de la végétation climacique et paraclimacique de l'Andalusie (Espagne). Ecologia Mediterranea, 10(1-2): 271-304.

GALIANO, E. -1960- Mapa de vegetación de la provincia de Jaén (mitad oriental). Instituto de Estudios Giennenses. Jaén.

LÓPEZ GONZÁLEZ, G. -1976- Contribución al conocimiento fitosociológico de la Serranía de Cuenca. I. Anales Inst. Bot. Cavanilles, 33: 587.

MARTÍNEZ PARRAS, J.M. y PEINADO, M. - 1987 Andalucía Oriental. In Peinado y Rivas Martínez (Eds.) La vegetación de España. Serv. Public. Univ. de Alcalá.
VALLE, F. - 1985- La vegetación del Macizo SeguraCazorla (Jaén). Anuario del adelantamiento de Cazorla, 26-27: 113-128.

GOMEZ MERCADO, F. y VALLE, F. -1988- Notas biogeográficas y ecológicas sobre el Macizo Cazorla-Segura: las bojedas. Actes del Simposi Internac. de Botánica Pius Font i Quer. Vol. II. Fanerogàmia: 271-276.

Aceptado para su publicación en Febrero de 1996

Dirección de los autores: Departamento de Biología Animal, Vegetal y Ecología. Facultad de C. Experimentales. Universidad de Jaén. Paraje Las Lagunillas, s/n, 23071. Jaén. 